\title{
Decreased EEG synchronization in Alzheimer's disease and mild cognitive impairment
}

\author{
T. Koenig $^{\mathrm{a}^{*}}$, L. Prichep ${ }^{\text {b,c }}$, T. Dierks ${ }^{\mathrm{a}}$, D. Hubl ${ }^{\mathrm{a}}$, L.O. Wahlund ${ }^{\text {d }}$, E.R. John ${ }^{\text {b,c }}$, \\ V. Jelic ${ }^{\text {d }}$
}

a) Department of Psychiatric Neurophysiology, University Hospital of Clinical Psychiatry Bern, Switzerland,

b) Brain Research Laboratories, NYU School of Medicine, New York, NY, USA

c) Nathan S. Kline Psychiatric Research Institute, Orangeburg, NY, USA

d) Division of Geriatric Medicine, Department of Clinical Neuroscience and Family Medicine, Karolinska Institute, Stockholm, Sweden

Corresponding author:

Dr. Thomas Koenig

Dept. of Psychiatric Neurophysiology

University Hospital of Clinical Psychiatry

Bolligenstrasse 111

CH-3000 BERN 60, Switzerland

Tel +41(31) 9309369

Fax +41(31) 9309961

E-mail (preferred): thomas.koenig@puk.unibe.ch 


\begin{abstract}
The hypothesis of a functional disconnection of neuro-cognitive networks in patients with mild cognitive impairment (MCI) and Alzheimer Dementia was investigated using baseline resting EEG data. EEG databases from New York (264 subjects) and Stockholm (155 subjects), including healthy controls and patients with varying degrees of cognitive decline or Alzheimer Dementia were analyzed using Global Field Synchronization (GFS), a novel measure of global EEG synchronization. GFS reflects the global amount of phase-locked activity at a given frequency by a single number; it is independent of the recording reference and of implicit source models. Patients showed decreased GFS values in Alpha, Beta and Gamma frequency bands, and increased GFS values in the Delta band, confirming the hypothesized disconnection syndrome. The results are discussed within the framework of current knowledge about the functional significance of the affected frequency bands.
\end{abstract}

Keywords: Alzheimer's Disease, Mild Cognitive Impairment (MCI), EEG, Synchronization, Binding, State Marker Trait Marker 


\section{Introduction}

Selective distribution of characteristic neurofibrillary pathology in Alzheimer's disease $(\mathrm{AD})$ follows the projection of anatomico-functional circuits of the brain ${ }^{[2,3]}$. During the course of the disease, the degenerative process spreads in a characteristic manner starting in the entorhinal region and progressing through the hippocampus and the limbic structures towards the neocortical association areas ${ }^{[2]}$. The disconnection is caused by the degeneration of the large cortical pyramidal neurons ${ }^{[14,16]}$ and assumingly occurs at the level of cortico-cortical projections. These cortico-cortical projections are crucial for higher order information processing, since they permit the integration of multimodal information through the coherent firing of extended neural networks. Therefore, Morrison ${ }^{[27]}$ suggested a "disconnection syndrome" as a model for the AD symptomatology. Since coherent firing of a sufficiently large number of neurons produces a detectable voltage field on the scalp, it is plausible that EEG data can be used to study changes in synchronization of neural circuits in AD that possibly occur at an early stage of the disease.

Within this context, a novel method called Global Field Synchronization (GFS) has recently been introduced to measure functional synchronization in frequency-domain EEG data ${ }^{[22]}$. For the entire set of EEG channels and for a given frequency, a single GFS value is obtained representing the percentage of EEG activity that oscillated with a common phase. High GFS values in a given frequency indicate therefore that a large part of the recorded brain activity at that frequency is phase locked. The presence of phase locked, oscillatory brain activity has been related to short-term binding of neural networks and may play an important role for higher order information processing and cognition ${ }^{[13,33]}$. Since resting EEG usually is not assumed to originate from very focal sources, the amount of synchronization measured by GFS can 
reasonably be assumed to be mediated thru a network of cortico-cortical connections. Contrary to coherence, where the stability of the phase relation between two preselected electrodes across analysis epochs is assessed and usually taken as indicator of cooperativity between the underlying regions, GFS makes no assumption about the spatial location of the activity. The computation of GFS assumes (in agreement with the existing volume conductor models of the head) that most types of measurable brain activity generate signals that are distributed over many electrodes on the scalp. If these signals show a predominant phase across all electrodes, this indicated that the (possibly many) local brain processes are interacting such that they appear to constitute a phase-locked, single and global meta-process. Compared to coherence that is local in space and global in time (i.e. requires selected pairs of electrodes recorded against a third, also pre-selected electrode, and a series of analysis epochs), GFS is global in space and local in time (i.e. it considers, during a single analysis epoch, all electrodes simultaneously and independent of the reference electrode). Therefore, since GFS does not require any assumptions about the location of the active processes, and since it can be assessed within relatively short (1-2 second) time periods, it may be well suited to study a hypothesized general disruption of shortlasting functional binding between extended neural networks. Additionally, the frequency information provided by the measure may be used to gain further information about the functional significance of the assumingly disrupted processes. Although animal work has shown that gamma $(40 \mathrm{~Hz})$ activity plays an important role for feature binding ${ }^{[8]}$ EEG research has indicated that other frequency bands are also crucially involved in human information processing ${ }^{[9]}$. A previous study on GFS comparing acutely schizophrenic patients with healthy controls has shown that patients had reduced GFS values in the theta band, which has been related to the 
existing hypothesis of a disconnection of working memory processes in schizophrenia [22].

The aim of the present study was to explore and extend the hypothesis of functional disconnection in $\mathrm{AD}$ and mild cognitive impairment (MCI) as reflected in GFS. The study was based on two independent, large samples encompassing healthy subjects, individuals with mild cognitive impairment and $\mathrm{AD}$ patients in different stages of the disease.

\section{Methods}

\subsection{Data collection in New York}

Subjects: The first group of patients was selected from consecutive, community residing elderly persons 50 to 89 years of age from the New York area, presenting with self-report of decline in cognitive functioning. Control subjects were recruited among healthy friends and family members of the patients. Medical, neurological, psychiatric and neuropsychological evaluations were conducted at the New York University School of Medicine (NYUSM), Aging and Dementia Research Center to exclude patients with conditions other than age-associated memory deficits or AD, which might interfere with, or confound the assessment of cognitive functioning. These exclusion criteria were: (a) past history of significant head trauma, seizures, mental retardation, or neurologic or psychiatric disorder other than AD; (b) any focal signs of significant neuropathology; (c) a diagnosis of multi-infarct dementia based on a history of cerebral infarction or transient ischemic attacks including any patients with a modified Hachinski score greater than or equal to $4^{[34]}$; (d) significant history of alcohol or drug abuse; (e) previous history of schizophrenia, major affective 
disorder, including any subjects with Hamilton Depression Scale (HAM-D) scores of 16 or greater; (g) cardiac, pulmonary, vascular, metabolic, or hematologic conditions of sufficient severity to adversely affect cognition or functioning and (h) other physical impairment of sufficient severity to adversely affect cognition or functioning. All included subjects discontinued any psychotropic or other cognitive-acting medication at least 2 weeks prior to the evaluation period.

Assessment of cognitive deterioration: All patients were assessed for the magnitude of cognitive decline staged from 1 to 6 on the Global Deterioration Scale (GDS) for age-associated cognitive decline and primary degenerative dementia ${ }^{[30]}$. Using this staging procedure, control subjects are assigned to GDS stage 1 and have neither subjective nor objective evidence for cognitive impairment, subjects at GDS stage 2 have subjective cognitive deficits in the absence of objective impairment, subjects at GDS stage 3 have objective evidence of memory deficit sufficient to interfere with performance in demanding employment and social settings, and subjects at GDS stages 4 to 7 have clear evidence of cognitive deficit comparable with mild (GDS=4), moderate $(\mathrm{GDS}=5)$, and moderately severe $(\mathrm{GDS}=6)$ AD. Stage specific relationships between GDS level assignments and scores on mental status, psychometric, and neuro-imaging measures have been reported ${ }^{[30]}$ and current data on the validity and reliability of this staging procedure has been reviewed ${ }^{[31]}$.

Following these evaluative procedures, the subjects were referred for EEG recording. The successfully recorded population consisted of normal elderly subjects (GDS=1; 25 subjects, mean age 67.1 years), patients with subjective complaints of cognitive impairment (GDS=2; 40 subjects, mean age 69.1 years), patients with mild cognitive impairment (GDS=3; 51 subjects, mean age 70.7 years), and patients with more severe deterioration associated with $\mathrm{AD}(\mathrm{GDS}=4 ; 71$ subjects, mean age 70.9 years; 
GDS $=5 ; 56$ subjects, mean age 71.7 years and GDS $6=21$ subjects, mean age 74.3 years), making a total of 264 subjects included in the analysis. Diagnosis of probable AD was based on DSM-IIIR ${ }^{[1]}$. There were no significant differences in age between any group of patients or controls. All subjects or legal representatives gave their written informed consent before participating in the study (as approved by NYUSM institutional review board).

EEG recording: Twenty minutes of eyes closed awake resting digital EEG data were collected from the 19 electrode sites of the International 10/20 System, referred to linked earlobes, while the subjects were seated comfortably in a light and sound attenuated room. The EEG was monitored throughout the recording for drowsiness and the patient was alerted if observed. Amplifiers had a bandpass from 0.5 to $70 \mathrm{~Hz}$ (-3dB point). A differential eye channel placed on the outer left canthus was used to detect eye movements. All electrode impedances were below $5 \mathrm{kOhms}$, the sampling rate was $200 \mathrm{~Hz}$. From the digital EEG, artifact-free epochs with a minimal duration of 2.5 seconds were selected offline by an automatic artifact rejection algorithm (based on channel-wise absolute amplitude thresholds) augmented by visual inspection by electrophysiologically expert editors. These editors eliminated any EOG, EMG, ECG or lateral eye-movement eluded by the automatic algorithm. The artifact exclusion procedure has been described and validated elsewhere ${ }^{[21]}$. The minimal total amount of available artifact free data was 1 minute per subject.

\subsection{Data collection in Stockholm}

Subjects: The second group of subjects was recruited among patients that were consecutively referred between 1995 and 1999 to the in- or out-patient Department of the Geriatric Clinic, Huddinge University Hospital, Stockholm, Sweden, for memory problems and investigation for suspected dementia. Sources of referral included 
general practitioners, neurologists, specialists of occupational medicine, or individuals seeking help directly because of self-experienced memory problems. Control subjects were recruited among the healthy family members of the patients and among healthy subjects recruited for a Driving and Aging project. All subjects underwent a careful physical, psychiatric and neurological examination as well as detailed neuropsychological assessment. Exclusion criteria were evidence of previous head trauma, previous infectious disease of the central nervous system, psychiatric illness or medication with psychotropic drugs and severe somatic diseases that could influence cognitive performance of the subjects. In addition, all patients performed MRI investigations to rule out eventual other causes of dementia like brain tumors, normotensive hydrocephalus, as well as SPECT investigations to support clinical diagnosis of $\mathrm{AD}$ by a typical finding of a reduced temporo-parietal cerebral blood flow ${ }^{[5,35]}$.

EEG recordings in $\mathrm{AD}$ patients were performed before treatment with any psychoactive or cognition enhancing drug had been initiated. The study was approved by the local ethical committee.

Assessment of cognitive deterioration: The global level of cognitive functioning of all subjects was assessed using the Mini Mental State Examination (MMSE) ${ }^{[10]}$ and the Full Scale IQ scores based on the four Wechsler subtests (FSIQ) ${ }^{[36]}$. The diagnosis of dementia was based on DSM-III-R criteria ${ }^{[1]}$, and NINCDS-ADRDA criteria ${ }^{[26]}$ served to establish a diagnosis of probable AD. The subjects were divided into the following 5 groups: a) healthy subjects with no signs of memory impairment (Controls; $n=21$, mean age 63.4 years), b) subjects who reported memory loss, but scored within 1 standard deviation (SD) from the age norm on the neuropsychological tests performed (Subjective memory impairment; $\mathrm{n}=30$, mean age 58.3 years), c) 
subjects who scored between 1 and 2 SD below the age norm on the neuropsychological tests, but did not fulfill diagnostic criteria for dementia (Objective memory impairment; $n=41$, mean age 61.0 years), d) patients meeting the criteria for dementia (DSM-III-R) and AD (NINCDS-ADRDA) with MMSE score of at least 20 (Mild $\mathrm{AD} ; \mathrm{n}=45$, mean age 62.0 years), and e) patients meeting the criteria for dementia (DSM-III-R) and Alzheimer's disease (NINCDS-ADRDA) with MMSE score between 15 and 20 (Moderate $A D ; n=18$, mean age 63.0). The successive groups were considered equivalent to the New York groups GDS 1 through 5. The total number of subjects was therefore 155 subjects, in an ANOVA, there was no significant effect of diagnostic group on age. Since the data collection in Stockholm had an emphasis on early detection these subjects in Stockholm were in the average 9.3 years younger than the subjects from New York.

EEG recording: Eyes closed resting EEG was recorded from 19 scalp electrodes applied according to the standard international 10/20 system. The EEG was recorded against linked ear lobes, band pass filtered between 1 and $30 \mathrm{~Hz}$ and digitized at 128 Hz. Artifact free 2 second epochs were selected by visual inspection, and at least 20 seconds of artifact free EEG data were available for each subject. This differed form the data collected in New York, but since later, GFS was computed separately in each epoch and averaged across epochs, and since it has been shown that $20 \mathrm{sec}$ of EEG are sufficient to reduce adequately the EEG variability ${ }^{[11]}$, this was not considered to be a problem for the reproducibility of results between the two centers.

\subsection{Group labeling}

For issues of clarity, a common labeling of the various degrees of cognitive decline and AD was used for both study centers: Controls (GDS 1 in New York, Controls in Stockholm), subjective memory impairment (GDS 2 in New York, subjective memory 
impairment in Stockholm), mild cognitive impairment (GDS 3 in New York, objective memory impairment in Stockholm), mild AD (GDS 4 in New York, mild AD in Stockholm), moderate AD (GDS 5 in New York, moderate AD in Stockholm), and moderately severe AD (GDS 6 in New York, not available in Stockholm).

\subsection{GFS Computation}

The computation of GFS has been described in detail in Koenig et al. ${ }^{[22]}$; and is reviewed here: At a given frequency, GFS measures the relative presence or absence of a common phase over all electrodes. For its computation, each EEG epoch is frequency transformed using the Fast Fourier Transformation (FFT). For each frequency, the real and complex parts of the signal of one electrode constitute a twodimensional vector; where origin of the vector represents the reference electrode, the length of the vector indicates the power of the signal and the direction of the vector represents the phase. If the signals from all electrodes would be in perfect phase or counter-phase, the vectors representing the signals would all point in the same or opposite direction and the endpoints of the vectors would thus lie on a straight line. If there would be no common phase, the vectors would point into different directions, and the endpoints of the vectors would be scattered. In order to determine how much the endpoints of the vectors approximate a straight line, they are entered into a twodimensional principal component (PC) analysis: The more the eigenvalue of the first PC exceeds the eigenvalue of the second PC, the more the endpoints approximate a straight line. If the first and the second PC are nearly equal, the vectors' end points are scattered. The GFS value is thus determined as that percentage of the total power that can maximally be explained by a single phase ${ }^{[22]}$. GFS ranges from 0 to 1 , high GFS values indicate the presence of a single, predominant phase over all electrodes, 
whereas low GFS indicate the absence of such a common phase. GFS is independent of the total power of the data and of the recording reference.

In the present data, GFS values were computed in each 2 sec EEG epoch and averaged separately for each frequency band (Delta: 1-4 Hz, Theta: 4.5-7.5Hz, Alpha: 8-12Hz, Beta $12.5-30 \mathrm{~Hz}$ and only for the dataset from New York Gamma: 35-45 Hz) within each subject. The software used for computing GFS is freely available at www.puk.unibe.ch/tk2/tk.htm (follow the 'Software' link).

\subsection{Statistical analysis}

Statistical analysis was based on single factor analyses of variance (ANOVAs) of GFS in each frequency band, using the degree of impairment as an independent variable. The datasets from the two recruitment sites were analyzed separately. In those frequency bands where significant group effects were found, the differences between groups were assessed using Fisher's test for post hoc comparisons. The level of significance was set at $5 \%$.

\section{Results}

The GFS means and SD for the different patient groups, frequency bands and recruitment sites are shown in Fig. 1.

Figure 1 about here

The ANOVAs on GFS as functions of the degree of impairment showed significant effects in the Delta band only in the data set from New York $(F(5,258)=2.52$, 
$p=0.030)$. In the Theta band, no effects were found in both recruitment sites. The Alpha band showed highly significant effect in both recruitment sites (Stockholm: $F(4,148)=7.81, p<0.0001$; New York: $F(5,258)=8.83, p<0.0001)$. In the Beta band, there was a significant effect in the dataset from New York $(F(5,258)=5.70$, $p<0.0001$ ) and a similar effect (although not reaching significance) in Stockholm $(F(4,148)=2.194, p=0.072)$. No significant effects were found in the Gamma band (only computed in the New York data). The results of the post-hoc comparisons are summarized in Table 1.

Although in the Gamma band, the ANOVA over all subject groups from New York was not significant, Gamma band GFS values were lower in all patient groups patients compared to controls (Fig. 1). Therefore, a single, two-tailed t-test was computed comparing Gamma band GFS between control subjects and with any degree of impairment. (This is equivalent to a single factor ANOVA comparing controls and patients). This t-test was significant $(t(262)=2.33, p=0.02)$.

Table 1 about here

Table 2 about here

\section{Discussion}

The analysis of GFS in elderly patients with various degrees of cognitive decline indicated a generally reduced amount of synchronized brain electric activity compared with normal controls. This finding is consistent with earlier EEG studies using coherence between pairs of electrodes ${ }^{[20,24,25]}$. However, the assumptions underlying the present results and the results obtained with coherence differ considerably: The 
coherence studies contain an implicit local source model by assuming that the signal recorded at one electrode represents only the activity underneath that electrode and not activity from other sources. Due to volume conduction and tangentially oriented generators, this is generally not the case. On the other hand, GFS measures synchronization on a spatially global level, where the neural activity oscillating with a common phase is considered as a single process, no matter where these neurons were distributed. Therefore, the computation of GFS implies no source-localization model. Another major difference is the temporal extend of the measures: Coherence measures the stability of phase relations over many epochs and therefore requires the signals to be stationary over these epochs. GFS assumes the signals to be stationary only within a single, short EEG epoch. Whereas coherence is thus local in space, but global in time, GFS is global in space and local in time.

The relation between GFS and degree of cognitive decline was similar for both recruitment sites, and dependent on frequency bands. The most pronounced effects were found in the alpha band. There was an almost continuous decrease of GFS with increasing cognitive impairments in the New York group, and in the Stockholm data set, the patients with a diagnosis of probable AD had lower GFS values compared to all other groups. This finding is interesting for several reasons: First, in normal controls, the GFS values were highest in the alpha band, and it may therefore be that neurodegenerative processes affect neural synchronization first in this frequency band. Second, alpha activity in normal subjects originates predominantly in posterior regions, where other imaging modalities have reported the first functional changes in $\mathrm{AD}^{[15,29]}$. Furthermore, in $\mathrm{AD}$ patients, the location of alpha sources correlated positively with brain glucose metabolism as assessed using positron emission tomography ${ }^{[6]}$. These findings suggest that a usually well-organized alpha activity 
disintegrates in time and changes in space with progressive deterioration in $\mathrm{AD}$ patients, independent of the other bands. Furthermore, these findings suggest that theta power abnormalities reported in QEEG studies ${ }^{[28]}$ as a correlate of cognitive deterioration may reflect a process that is independent from the reported GFS deviations.

The results found in the beta band were similar to those in the alpha band showing decreased synchronization in more impaired subjects. The beta band has classically been related to excitatory activity and cognitive processes that deteriorate during AD. Furthermore, beta spectral power has also been shown to decrease in $\mathrm{AD}^{[17]}$.

In the patient groups, GFS values were also found to be decreased in the gamma band. This effect might either be a continuation of the effects seen in the beta band or it may relate to the $40 \mathrm{~Hz}$ activity described in animal studies which is involved in the establishment of synchrony among widely distributed neurons ${ }^{[23]}$. However, since GFS values in the Gamma band showed a much larger inter-individual variance compared to the other frequency bands, and since it did not differentiate between the patient groups, its relevance for EEG synchronization in AD remains open.

In the delta band, GFS was selectively increased in the most impaired patients (patients with GDS $=6$; these patients were only present in the data-set from New York). This apparently contradictory finding could possibly be explained by the progressive cortical cholinergic deafferentation observed in $\mathrm{AD}^{[12]}$. In animal lesion studies, increased delta activity has been reported after destruction of the Nucleus Basalis ${ }^{[4]}$, the main production site of acetylcholin ${ }^{[32]}$. In humans, it has also been demonstrated that delta abnormalities occur only in the later stages of deterioration. Furthermore, treatment response after long-term therapy with acetylcholinesterase inhibitors in AD patients showed reduction in delta and theta activity ${ }^{[18]}$. 
The present results are therefore considered to be compatible with the hypothesis of a neurofunctional disconnection in $\mathrm{AD}$. However, although the results from the two datasets gave converging support for this hypothesis, there are also important differences between the datasets that need to be mentioned. In the dataset from Stockholm, there were nearly no differences in alpha GFS between healthy controls and subjects with subjective memory impairments or mild cognitive impairment. In the dataset from New York however, alpha and beta band GFS decreased nearly continuously with increasing impairment. There were nearly significant GFS differences already between controls and patients with mild cognitive impairment in the alpha band, and even significant differences between controls and patients with subjective memory impairment in the beta band. It is thus not clear whether the GFS differences described here can be used as state marker of cognitive decline (as the data from New York suggests) or as trait marker of AD (what the data from Stockholm suggests), and what role the different assessment systems played in this context.

In general, the method seems to be promising for further EEG research, since its computation relies on few assumptions that allow a comprehensive interpretation and since its global characteristics simplify statistics. Furthermore, it would be of interest to investigate whether previously reported differences in EEG coherence between AD and other typed of dementia (i.e vascular dementia ${ }^{[24]}$ ) are similarly reflected in GFS. Interestingly, differential decrease in GFS has been demonstrated in our recent study where patients with frontal lobe dementia (FLD) were compared to AD patients and healthy individuals ${ }^{[19]}$, where FLD patients had significantly lower GFS in all frequency bands when compared to both $\mathrm{AD}$ patients and healthy elderly controls. Future correlative studies with functional and structural neuroimaging methods and 
indices of neuropsychological performance in patients with dementia should thus add to the understanding of the functional and behavioral relevance of GFS.

Acknowledgements: The study was supported by the Gamla Tjänarinnor Foundation, by the Loo and Hans Ostermann's Foundation and the Alzheimer Foundation.

The NYUSM data were collected as part of an ongoing collaboration between the New York Aging and Dementia Research Center under the direction of Drs. Steven Ferris and Barry Reisberg and the Brain Research Laboratories of NYU School of Medicine. 


\section{References}

[1] American Psychiatric Association. Diagnostic and Statistical Manual of Mental Disorders (DSM-III-R). Washington, DC: American Psychiatric Association, 1987.

[2] Braak H, Braak E. Neuropathological stageing of Alzheimer-related changes. Acta Neuropathol (Berl) 1991; 4(82):239-59.

[3] Brun A, Englund E. Regional pattern of degeneration in Alzheimer's disease: neuronal loss and histopathological grading. Histopathology 1981; 5(5):549-64.

[4] Buzsaki G, Bickford RG, Ponomareff G, Thal LJ, Mandel R, Gage FH. Nucleus basalis and thalamic control of neocortical activity in the freely moving rat. J Neurosci 1988; 11(8):4007-26.

[5] Claus JJ, van Harskamp F, Breteler MM, Krenning EP, de K, I, van der Cammen TJ, Hofman A, Hasan D. The diagnostic value of SPECT with Tc 99m HMPAO in Alzheimer's disease: a population-based study. Neurology 1994; 3 Pt 1(44):454-61.

[6] Dierks T, Ihl R, Frolich L, Maurer K. Dementia of the Alzheimer type: effects on the spontaneous EEG described by dipole sources. Psychiatry Res $1993 ; 3(50): 151-62$.

[7] Dierks T, Jelic V, Pascual-Marqui RD, Wahlund L, Julin P, Linden DE, Maurer K, Winblad B, Nordberg A. Spatial pattern of cerebral glucose metabolism (PET) correlates with localization of intracerebral EEGgenerators in Alzheimer's disease. Clin Neurophysiol 2000; 10(111):1817-24.

[8] Engel AK, Singer W. Temporal binding and the neural correlates of sensory awareness. Trends Cogn Sci 2001; 1(5):16-25.

[9] Fernandez T, Harmony T, Rodriguez M, Bernal J, Silva J, Reyes A, Marosi E. EEG activation patterns during the performance of tasks involving different components of mental calculation. Electroencephalogr Clin Neurophysiol 1995; 3(94):175-82.

[10] Folstein MF, Folstein SE, McHugh PR. "Mini-mental state". A practical method for grading the cognitive state of patients for the clinician. $\mathrm{J}$ Psychiatr Res 1975; 3(12):189-98.

[11] Gasser T, Bacher P, Steinberg H. Test-retest reliability of spectral parameters of the EEG. Electroencephalogr Clin Neurophysiol 1985; 4(60):312-9. 
[12] Geula C. Abnormalities of neural circuitry in Alzheimer's disease: hippocampus and cortical cholinergic innervation. Neurology 1998; 1 Suppl 1(51):S18-S29.

[13] Gray CM. Synchronous oscillations in neuronal systems: mechanisms and functions. J Comput Neurosci 1994; 1-2(1):11-38.

[14] Hardy JA, Mann DM, Wester P, Winblad B. An integrative hypothesis concerning the pathogenesis and progression of Alzheimer's disease. Neurobiol Aging 1986; 6(7):489-502.

[15] Haxby JV, Grady CL, Duara R, Schlageter N, Berg G, Rapoport SI. Neocortical metabolic abnormalities precede nonmemory cognitive defects in early Alzheimer's-type dementia. Arch Neurol 1986; 9(43):882-5.

[16] Hof PR, Bouras C. Object recognition deficit in Alzheimer's disease: possible disconnection of the occipito-temporal component of the visual system. Neurosci Lett 1991; 1(122):53-6.

[17] Holschneider DP, Leuchter AF. Beta activity in aging and dementia. Brain Topogr 1995; 2(8):169-80.

[18] Jelic V, Dierks T, Amberla K, Almkvist O, Winblad B, Nordberg A. Longitudinal changes in quantitative EEG during long-term tacrine treatment of patients with Alzheimer's disease. Neurosci Lett 1998; 2(254):85-8.

[19] Jelic V, Koenig T, Lindau M, Dierks T, Wahlund LO. EEG amplitude and synchronisation distinguish Alzheimer's disease, frontotemporal dementia and normal aging. Neurobiol Aging 2002; (23 [1Suppl]):S355.

[20] Jelic V, Shigeta M, Julin P, Almkvist O, Winblad B, Wahlund LO. Quantitative electroencephalography power and coherence in Alzheimer's disease and mild cognitive impairment. Dementia 1996; 6(7):314-23.

[21] John ER, Prichep LS, Easton P. Normative data banks and Neurometrics: Basic concepts, methods and results of norm construction. In: Gevins AS, Remond A, editors. Handbook of Electroencephalography and Clinical Neurophysiology. Amsterdam: Elsevier, 1987.

[22] Koenig T, Lehmann D, Saito N, Kuginuki T, Kinoshita T, Koukkou M. Decreased functional connectivity of EEG theta-frequency activity in first-episode, neuroleptic-naive patients with schizophrenia: preliminary results. Schizophr Res 2001; 1-2(50):55-60.

[23] Konig P, Engel AK, Singer W. Relation between oscillatory activity and longrange synchronization in cat visual cortex. Proc Natl Acad Sci USA 1995; 1(92):290-4. 
[24] Leuchter AF, Newton TF, Cook IA, Walter DO, Rosenberg-Thompson S, Lachenbruch PA. Changes in brain functional connectivity in Alzheimer-type and multi- infarct dementia. Brain 1992; (115):154361.

[25] Locatelli T, Cursi M, Liberati D, Franceschi M, Comi G. EEG coherence in Alzheimer's disease. Electroencephalogr Clin Neurophysiol 1998; 3(106):229-37.

[26] McKhann G, Drachman D, Folstein M, Katzman R, Price D, Stadlan EM. Clinical diagnosis of Alzheimer's disease: report of the NINCDSADRDA Work Group under the auspices of Department of Health and Human Services Task Force on Alzheimer's Disease. Neurology 1984; 7(34):939-44.

[27] Morrison JH, Rogers J, Scherr S, Levis DA, Campbell MJ, Bloom FE, Benoit $\mathrm{R}$. The laminar and regional distribution of neocortical somatostatin and neuritic plaques: Implications for Alzheimer's Disease as a global neocortical disconnection syndrome. In: Scheibel AB, Wechsler AF, editors. The Biological Substrates of Alzheimer's Disease. Orlando: Academic Press, 1996.

[28] Prichep LS, John ER, Ferris SH, Reisberg B, Almas M, Alper K, Cancro R. Quantitative EEG correlates of cognitive deterioration in the elderly. Neurobiol Aging 1994; 1(15):85-90.

[29] Rapoport SI. Discriminant analysis of brain imaging data identifies subjects with early Alzheimer's disease. Int Psychogeriatr 1997; (9 Suppl 1):229-35.

[30] Reisberg B, Ferris SH, de Leon MJ, Crook T. Global Deterioration Scale (GDS). Psychopharmacol Bull 1988; 4(24):661-3.

[31] Reisberg B, Ferris SH, de Leon MJ, Sinaiko E, Franssen E, Kluger A, Mir P, Borenstein J, George AE, Shulman E, Steinberg E, Cohen J. Stagespecific behavioral cognitive and in vivo changes in community residing subjects with Age-Associated Memory Impairment and Primary Degenerative Dementia of the Alzheimer type. Drug Develop Research 1988; 15101-14.

[32] Riekkinen P, Jr., Sirvio J, Riekkinen P. Relationship between the cortical choline acetyltransferase content and EEG delta-power. Neurosci Res 1990; 1(8):12-20.

[33] Roelfsema PR, Engel AK, Konig P, Singer W. Visuomotor integration is associated with zero time-lag synchronization among cortical areas. Nature 1997; 6612(385):157-61.

[34] Rosen WG, Terry RD, Fuld PA, Katzman R, Peck A. Pathological verification of ischemic score in differentiation of dementias. Ann Neurol 1980; $5(7): 486-8$. 
Decreased EEG synchronization in Alzheimer's disease and mild cognitive impairment

[35] Waldemar G. Functional brain imaging with SPECT in normal aging and dementia. Methodological, pathophysiological, and diagnostic aspects. Cerebrovasc Brain Metab Rev 1995; 2(7):89-130.

[36] Wechsler D. WAIS-R manual: Wechsler adult intelligence scale- revised. New York: Harcourt Brace Jovanovich, 1981. 


\section{Tables}

Table 1: Significant differences* in the frequency bands between the groups of healthy subjects and patients in different stages of the disease, New York data.

\begin{tabular}{|c|c|c|c|c|c|}
\hline Group & Delta & Theta & Alpha & Beta & Gamma \\
\hline C vs S & n.s. & - & n.s. & $* \downarrow$ & - \\
\hline $\mathrm{C}$ vs $\mathrm{O}$ & n.s. & - & $(* \downarrow)$ & $* \downarrow$ & - \\
\hline C vs Mild AD & n.s. & - & $* \downarrow$ & $* \downarrow$ & - \\
\hline C vs Mod AD & n.s. & - & $* \downarrow$ & $* \downarrow$ & - \\
\hline C vs Mod sev AD & $* \uparrow$ & - & $* \downarrow$ & $* \downarrow$ & - \\
\hline $\mathrm{S}$ vs $\mathrm{O}$ & n.s. & - & n.s. & n.s. & - \\
\hline S vs Mild AD & n.s. & - & $* \downarrow$ & $(* \downarrow)$ & - \\
\hline S vs Mod AD & n.s. & - & $* \downarrow$ & $* \downarrow$ & - \\
\hline S vs Mod sev AD & $* \uparrow$ & - & $* \downarrow$ & $* \downarrow$ & - \\
\hline O vs Mild AD & n.s. & - & $(* \downarrow)$ & $* \downarrow$ & - \\
\hline O vs Mod AD & n.s. & - & $* \downarrow$ & $* \downarrow$ & - \\
\hline $\mathrm{O}$ vs $\mathrm{M}$ sev $\mathrm{AD}$ & $* \uparrow$ & - & $* \downarrow$ & $* \downarrow$ & - \\
\hline Mild AD vs Mod AD & n.s. & - & $(* \downarrow)$ & n.s. & - \\
\hline Mild AD vs M sev AD & $* \uparrow$ & - & $* \downarrow$ & n.s. & - \\
\hline Mod AD vs $M$ sev AD & $* \uparrow$ & - & n.s. & n.s. & - \\
\hline \multicolumn{6}{|c|}{ subjective memory impairment, $\mathrm{O}$ - objective cognitive impairment, Mild AD - mild } \\
\hline \multicolumn{6}{|c|}{ Alzheimer's disease, Mod AD - moderate Alzheimer's disease, M sev AD - } \\
\hline moderately severe AD. & The $\mathrm{d} c$ & licates th & VA wa & gnific & it and no \\
\hline
\end{tabular}


Table 2: Significant differences* in the frequency bands between the groups of healthy subjects and patients in different stages of the disease, Stockholm data.

\begin{tabular}{lcccc}
\hline Group & Delta & Theta & Alpha & Beta \\
\hline C vs S & - & - & n.s. & n.s. \\
C vs O & - & - & n.s. & n.s. \\
C vs Mild AD & - & - & $* \downarrow$ & $* \downarrow$ \\
C vs Mod AD & - & - & $* \downarrow$ & $* \downarrow$ \\
S vs O & - & - & n.s. & n.s. \\
S vs Mild AD & - & - & $* \downarrow$ & $(* \downarrow)$ \\
S vs Mod AD & - & - & $* \downarrow$ & $* \downarrow$ \\
O vs Mild AD & - & - & $* \downarrow$ & n.s. \\
O vs Mod AD & - & - & $* \downarrow$ & n.s. \\
Mild AD vs Mod AD & - & - & n.s. & n.s. \\
\hline
\end{tabular}

$* \mathrm{p}<0.05 ;\left(^{*}\right) 0.05<\mathrm{p}<0.1$; Fisher's test for post-hoc comparisons.

$\mathrm{C}$ - controls, $\mathrm{S}$ - subjective memory impairment, $\mathrm{O}$ - objective cognitive impairment, Mild AD - mild Alzheimer's disease, mod AD - moderate Alzheimer's disease. The dash indicates the ANOVA was not significant and no post-hoc tests were computed, arrows indicate the direction of the change. 
Decreased EEG synchronization in Alzheimer's disease and mild cognitive impairment

\section{Figure legend}

Fig. 1: Means and standard deviations of the obtained GFS values (vertical axis) for the different frequency bands (horizontal axis). Increasing darkness of the bars indicate increased impairment. Upper graph: data from New York, lower graph: data from Stockholm. * indicates frequency bands were the ANOVA was significant, $(*)$ indicate tendencies below $\mathrm{p}<0.1$. 
Fig 1

New York Data

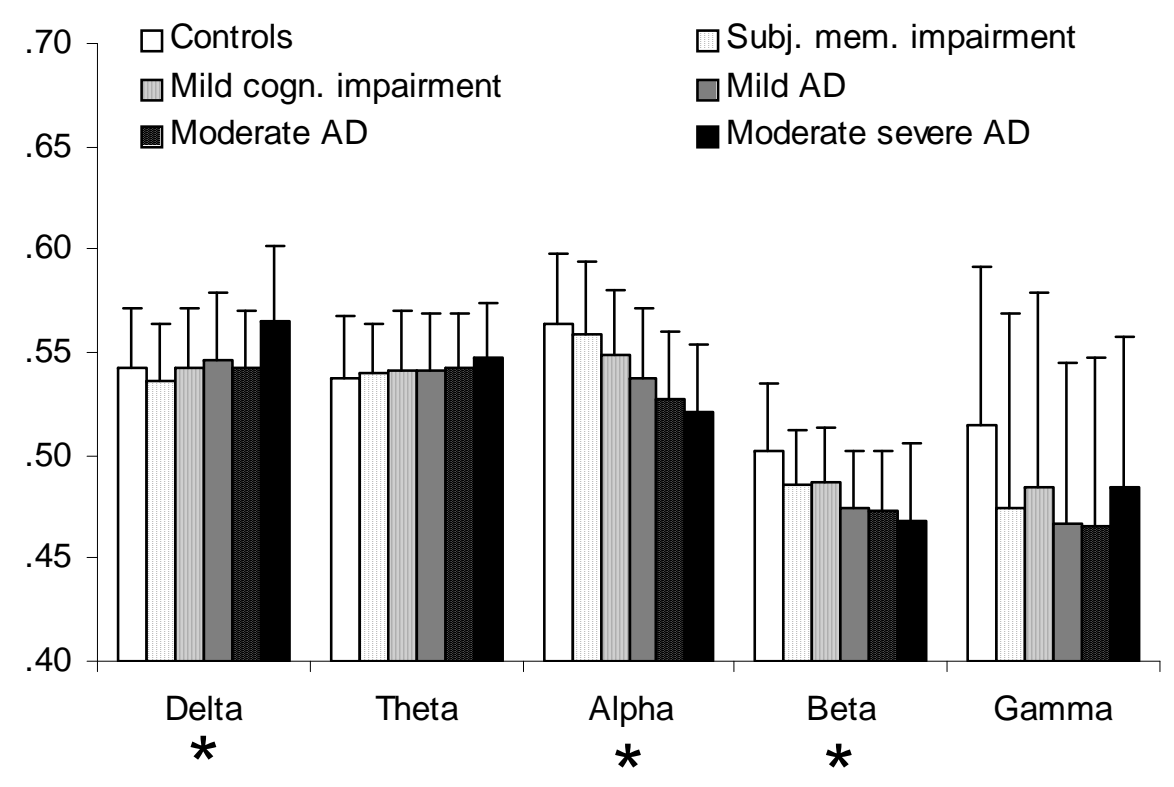

Stockholm Data

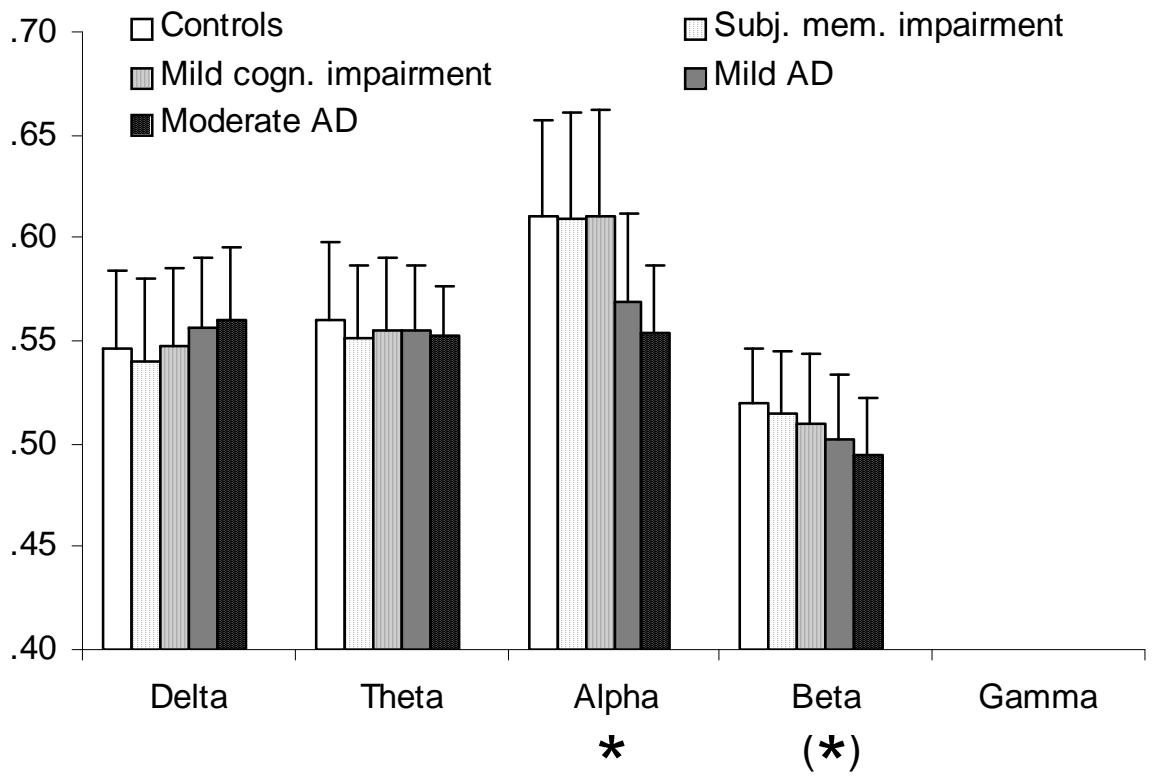

